\title{
Liquid crystalline states for two-dimensional electrons in strong magnetic fields
}

\author{
Orion Ciftja \\ Department of Physics, Prairie View A\&M University, Prairie View, Texas 77446, USA \\ Cintia M. Lapilli \\ Department of Physics and Astronomy, University of Missouri-Columbia, Columbia, Missouri 65211, USA \\ Carlos Wexler \\ Department of Physics and Astronomy, University of Missouri-Columbia, Columbia, Missouri 65211, USA
}

(Received 6 November 2003; published 17 March 2004)

\begin{abstract}
Based on the Kosterlitz-Thouless-Halperin-Nelson-Young theory of two-dimensional melting and the analogy between Laughlin states and the two-dimensional one-component plasma, we investigate the possibility of liquid crystalline states in a single Landau level (LL). We introduce many-body trial wave functions that are translationally invariant but possess twofold (i.e., nematic), fourfold (tetratic), or sixfold (hexatic) broken rotational symmetry at respective filling factors $\nu=1 / 3,1 / 5$, and $1 / 7$ of the valence LL. We find that the above liquid crystalline states exhibit a soft charge-density wave (CDW) which underlies the translationally invariant state but which is destroyed by quantum fluctuations. By means of Monte Carlo simulations, we determine that, for a considerable variety of interaction potentials, the anisotropic states are energetically unfavorable for the lowest and first excited LL's (with index $L=0,1$ ), whereas the nematic is favorable at the second excited LL $(L=2)$.
\end{abstract}

DOI: 10.1103/PhysRevB.69.125320

PACS number(s): 73.43.-f, 73.20.Mf, 64.70.Md, 52.27.Aj

\section{INTRODUCTION}

In 1983 Laughlin $^{1}$ introduced his famous trial-wave function

$$
\Psi_{1 / m}=\prod_{i<j}^{N}\left(z_{i}-z_{j}\right)^{m} \exp \left\{-\frac{1}{4 l_{0}^{2}} \sum_{k=1}^{N}\left|z_{k}\right|^{2}\right\},
$$

to describe the fractional quantum Hall effect (FQHE) states $^{2-5}$ for filling factors $\nu=1 / m$ of the lowest Landau level (LLL), where $m$ is an odd integer. Immediately after this discovery, many attempts were done to compare the stability of these states against other known ground states, typically Wigner crystal (WC) states. ${ }^{6-8}$ At absolute zero $(T=0)$ the current theoretical understanding is that WC states are favorable for filling factors smaller than a critical value $\nu_{c}$ $\simeq 1 / 6.5 .^{7,8}$ For larger filling factors of the LLL, the electrons are believed to form a quantum liquid state with Laughlin wave function being an excellent choice for $\nu=1 / m$ (with $m=1,3,5) .{ }^{9}$ Because of its translational and rotational invariance, Laughlin's wave function can be used to describe a liquid state of the electrons in the LLL, as can be seen by writing $\left|\Psi_{1 / m}\right|^{2}$ as a classical distribution function ${ }^{10,11}$

$$
\begin{gathered}
\left|\Psi_{1 / m}\right|^{2} \propto e^{-\beta V}, \\
\text { where }-\beta V=2 m \sum_{i<j}^{N} \ln \left|z_{i}-z_{j}\right|-\frac{1}{2 l_{0}^{2}} \sum_{k=1}^{N}\left|z_{k}\right|^{2},
\end{gathered}
$$

and $V$ is the potential energy of a classical two-dimensional one-component-plasma (2DOCP) system. Using the formal analogy between the Laughlin wave function and the 2DOCP we can identify a dimensionless coupling constant, $\Gamma \equiv \beta e^{2}$ $=e^{2} /\left(k_{B} T\right)=2 m$. An equilibrium state of the 2DOCP is en- tirely characterized by $\Gamma$ and the freezing transition in this case was located at $\Gamma \approx 140 .{ }^{10}$ Employing the analogy between the temperature of the classical plasma and the filling factor of the LLL, we should expect a freezing transition as we decrease the electronic filling factor in the quantum Hall regime. Because of the different quantum nature of the electronic correlations in the FQHE, it was found that such a system is a Laughlin liquid for filling factors $\nu=1 / 3$ and $1 / 5$, but becomes crystal for filling factors smaller than $\nu_{c}$ $\approx 1 / 6.5$ (this value is about an order of magnitude larger than that deduced from the classical 2DOCP analogy).

It is feasible that, in analogy to the classical freezing transition realized by cooling down a $2 \mathrm{DOCP}$, the transition to a solid (WC) state obtained by reducing the filling factor in the electron case may be interpreted as a topological KosterlitzThouless-type transition. ${ }^{12}$ This would be the correlated electron system counterpart of the well-known 2D melting problem. Although the 2D melting is not fully understood, an elegant and reliable theory of melting has been proposed in the 1970s by Kosterlitz, Thouless, Halperin, Nelson, and Young (KTHNY). ${ }^{12-14}$ The KTHNY theory predicts that an intermediate third phase called hexatic, will exist between the hexagonal solid and the liquid phases in a certain portion of the phase diagram (perhaps in a somewhat narrow range of temperatures). In the liquid phase there is no long-range translational or rotational order (the system is both translationally and rotationally invariant). In the solid phase the system has quasi-long-range translational and true longrange rotational order. The hexatic phase in the KTHNY theory is thought to have no true long-range translational order, but does retain quasi-long-range orientational order (the system is translationally invariant, but not rotationally invariant at least for short distances). The intermediate 
hexatic phase is often considered most important since it has a symmetry intermediate between the hexagonal solid and the liquid.

Recent experiments in very high mobility ( $\mu$ $\sim 10^{7} \mathrm{~m} / \mathrm{Vs}$ ) GaAs$/ \mathrm{Al}_{x} \mathrm{Ga}_{1-x}$ As heterostructures have shown a variety of low-temperature phases with exotic properties. Since 1999 it has been known that in transitional regions between $\mathrm{QH}$ plateaus for high LL's (with LL index $L \geqslant 2$ ) either a smectic or nematic phase exists. ${ }^{15-20}$ In fact, one of us calculated to a reasonable accuracy the anisotropicisotropic transition temperature as a topological process. ${ }^{17} \mathrm{In}$ 2002 a melting transition from the WC state to a FQHE-like state was observed at ca. $130 \mathrm{mK}$ (Ref. 21) and speculation mounted to suggest that possibly this transition occurs to a hexatic mesophase. ${ }^{22}$

On this grounds we investigate the possibility of various liquid crystalline mesophases in a partially filled LL. Given that two-dimensional liquid crystals may posses different forms of rotational group symmetry, we select a set of possible candidates, having $C_{2}$ (nematic), $C_{4}$ (tetratic), and $C_{6}$ (hexatic) rotational group symmetry (note that, in principle, higher symmetry groups are also possible for a liquid crystal, e.g., a liquid quasicrystal with a $C_{10}$ symmetry-we have not explored, however such possibilities in this paper ${ }^{23}$ ). Our results indicate that the states studied exhibit a soft chargedensity wave (CDW) which underlies the translationally invariant state but which is destroyed by quantum fluctuations. We perform Monte Carlo (MC) simulations and determine that for a wide range of interactions the anisotropic states are energetically unfavorable for the lowest and first excited LL's (with index $L=0,1$ ), whereas the nematic is favorable at the second excited LL $(L=2)$.

In Sec. II we describe the types of states that were considered for our calculations. Section III presents the types of interaction potential considered and explains the methods used to calculate the properties of the system. Section IV contains the results obtained and a discussion of their meaning. The underlying soft CDW is discussed in Sec. V. Finally, the conclusions are presented in Sec. VI.

\section{LIQUID CRYSTAL STATES}

In this paper we consider liquid crystalline phases with no translational order but with quasi-long-range orientational order with various rotational symmetry groups $C_{2}, C_{4}$, and $C_{6}$; corresponding to a nematic, tetratic, and hexatic phase, respectively. There are some basic requirements on how we construct these states: (i) the states must obey Fermi statistics, i.e., they must have odd parity under the exchange of any pair of electrons, (ii) the states must be translationally invariant (at least far away from the boundaries of the system in case of a finite number of electrons), (iii) there must be a broken rotational symmetry belonging to the proper symmetry group, (iv) the states must belong to a single LL to avoid the large cyclotron energy cost $\hbar \omega_{c}=\hbar e B / m_{e}$, where $B$ is the magnetic field, and $e$ and $m_{e}$ are the electron charge and mass, respectively (also note that as we will show later, various properties at any LL can be readily obtained from properties calculated in the LLL).
A class of such wave functions satisfying all these requirements are the so-called broken-rotational-symmetry (BRS) wave functions ${ }^{24,18-20,22}$ that are systematically constructed by properly splitting the zeros of the Laughlin liquid state [in essence, the idea is to place the vortices that perform the composite fermion $(\mathrm{CF})$ transformation ${ }^{4,5}$ around the location of the electron, rather "on top" of them]. Let us consider the Laughlin wave function as given in Eq. (1), where $z_{k}=x_{k}+i y_{k}$ is $k$ th electron position in the $x y$ plane in complex notation, and $l_{0}=[\hbar /(e B)]^{1 / 2}$ is the magnetic length. This wave function represents a gaped, uniform, and isotropic liquid, and is an excellent description of a liquid state at filling factor $\nu=1,1 / 3$, and $1 / 5$ of the LLL (for $\nu=1 / 7$, the WC state prevails, see previous discussion, and Ref. 22).

To build a liquid crystal (BRS) state out of the liquid states we split the zeros of the wave function in a way that conserves the antisymmetry (Fermi statistics) and translational invariance, but breaks the rotational invariance of the wave function. This is done by introducing a preferred set of directions ${ }^{24,18-20,22}$ into the wave function creating a degree of anisotropy. A generalized liquid-crystal wave function for a filling factor $\nu=1 / \mathrm{m}$ can then be easily written as

$$
\begin{aligned}
\Psi_{1 / m}^{\alpha}= & \left\{\prod_{i<j}^{N}\left[\prod_{\mu=1}^{m-1}\left(z_{i}-z_{j}-\alpha_{\mu}\right)\right]\right\} \prod_{i<j}^{N}\left(z_{i}-z_{j}\right) \\
& \times \exp \left\{-\frac{1}{4 l_{0}^{2}} \sum_{k=1}^{N}\left|z_{k}\right|^{2}\right\},
\end{aligned}
$$

where the complex directors $\alpha_{\mu}$ are distributed in pairs of opposite value in the complex plane (to satisfy Fermi statistics). In this paper we focus on the states with the highest level of discrete symmetry possible at each filling factor, which is set by distributing the $\alpha_{\mu}$ symmetrically in a circle around the origin,

$$
\alpha_{\mu}=\alpha e^{i 2 \pi(\mu-1) /(m-1)}, \quad \mu \in\{1,2, \ldots,(m-1)\} .
$$

Without loss of generality $\alpha$ can be taken to be real. The wave function in Eq. (3) represents a homogeneous liquid crystalline state at filling factors $\nu=1 / \mathrm{m}$, is antisymmetric, lies entirely in the LLL, and is smoothly connected to the isotropic Laughlin state for $\alpha=0$.

\section{INTERACTION POTENTIALS AND MONTE CARLO SIMULATION}

For our simulations we consider $N$ electrons in a charge neutralizing background. When considering the quantum Hamiltonian $\hat{H}=\hat{K}+\hat{V}$, the strong magnetic field quantizes the kinetic energy $\hat{K}$ so that single-LL wave functions have a constant (and thus irrelevant) kinetic energy, $\langle\hat{K}\rangle / N$. The only relevant contribution comes, therefore, from the total potential-energy operator

$$
\hat{V}=\hat{V}_{e e}+\hat{V}_{e b}+\hat{V}_{b b},
$$

consisting of electron-electron, electron-background and background-background, interactions. 
It has been a common practice to work on the surface of a sphere $^{5}$ in order to minimize boundary effects in the finitesize computations. However, due to the anisotropic nature of the states under consideration this scheme would produce significant problems due to the need to have topological defects at the "poles" of the sphere. We therefore work on a simpler disk geometry, where the neutralizing positive background has a uniform density $\rho_{0}=\nu /\left(2 \pi l_{0}^{2}\right)$ and is spread over a disk of radius $R_{N}=l_{0}(2 N / \nu)^{1 / 2}$ with an area $\Omega_{N}$ $=\pi R_{N}^{2}$.

Our goal is to thoroughly investigate the possibility of a liquid crystal state in the LLL for electrons interacting not only with the usual bare Coulomb potential $v_{C}\left(r_{12}\right)$ $=e^{2} /\left(\epsilon r_{12}\right)$ but also for a variety of other reasonable effective potentials that take into consideration the finite thickness of the quasi-2D electron layer. As previously shown by Zhang and Das Sarma (ZDS), ${ }^{27}$, the electron-electron interaction in a quasi-2D system can be written as

$$
\begin{aligned}
& v_{Z D S}\left(r_{12}\right)=\frac{e^{2}}{\epsilon} \int_{0}^{\infty} d q J_{0}\left(q r_{12}\right) F(q, b), \\
& F(q, b)=\left(1+\frac{9}{8} \frac{q}{b}+\frac{3}{8} \frac{q^{2}}{b^{2}}\right)\left(1+\frac{q}{b}\right)^{-3},
\end{aligned}
$$

where $r_{12}$ is the 2D distance separating the two electrons, $\epsilon$ is the average background dielectric constant, $J_{0}$ is the Bessel function of zeroth order, and $b$ is a parameter related to the finite thickness of the 2D layer (if we define the average thickness as $\bar{Z}$, then $b=3 / \bar{Z}$ ). In addition, we also consider two other interaction potentials

$$
\begin{gathered}
v_{1}\left(r_{12}\right)=\frac{e^{2}}{\epsilon} \frac{1}{\sqrt{r_{12}^{2}+\lambda^{2}}}, \\
v_{2}\left(r_{12}\right)=\frac{e^{2}}{\epsilon} \frac{1-\exp \left(-\frac{r_{12}}{\lambda}\right)}{r_{12}} .
\end{gathered}
$$

The two model potentials include the thickness effect phenomenologically ${ }^{27}$ through the length parameter, $\lambda=\bar{Z} / 2$ $=1.5 / \mathrm{b}$. All the above potentials have the same Coulomb behavior for large $r_{12}$, but differ from the bare Coulomb potential for small $r_{12}$.

To consider the zero-temperature stability of the liquidcrystal states of Eq. (3) with respect to the uniform isotropic liquid state counterparts, we performed extensive MC simulations in order to compute the energy and other quantities for the four different interaction potentials. Since the potentials involved are merely single- and two-body interactions, we need to accurately determine all single- and doubleparticle distribution functions, i.e., the density $\rho(\mathbf{r})$ $\equiv\left\langle\sum_{i=1}^{N} \delta\left(\mathbf{r}_{i}-\mathbf{r}\right)\right\rangle$, and the pair-correlation function $g\left(\mathbf{r}_{12}\right)$, respectively. The determination of such functions allows an accurate determination of all potential energies in the $N$

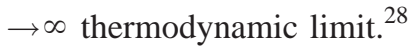

By definition, the pair-correlation function $g\left(\mathbf{r}_{12}\right)$ is the conditional probability [normalized so that $g(\infty)=1$ ] to find an electron at position $\mathbf{r}^{\prime \prime}$ given that another electron is found at position $\mathbf{r}^{\prime}=\mathbf{r}^{\prime \prime}-\mathbf{r}_{12}$,

$$
g\left(\mathbf{r}_{12}\right) \equiv \frac{1}{\rho_{0}^{2}}\left\langle\sum_{i \neq j}^{N} \delta\left(\mathbf{r}_{i}-\mathbf{r}^{\prime}\right) \delta\left(\mathbf{r}_{j}-\mathbf{r}^{\prime \prime}\right)\right\rangle,
$$

where $\rho_{0}=\nu /\left(2 \pi l_{0}^{2}\right)$ is the average bulk electron density. It is also useful to define the static structure factor $S(\mathbf{q})$, which is given by the 2D Fourier transform of $g\left(\mathbf{r}_{12}\right)$,

$$
S(\mathbf{q})-1=\rho_{0} \int d^{2} r_{12} e^{-i \mathbf{q} \cdot \mathbf{r}_{12}\left[g\left(\mathbf{r}_{12}\right)-1\right] .}
$$

Note that, because of the anisotropy of the wave function, both functions are explicitly angle dependent: $g\left(\mathbf{r}_{12}\right)$ $=g\left(r_{12}, \theta\right)$ and $S(\mathbf{q})=S\left(q, \theta_{q}\right)$ for $\alpha \neq 0$. It is also worth noting that the charge neutrality sum rule guarantees that $S(\mathbf{q}) \propto q^{2}$ for $q \rightarrow 0 . .^{10,25,26}$

In the thermodynamic limit, the ground-state correlation energy per particle can be easily computed from ${ }^{28}$

$$
E_{\alpha}=\frac{\langle\hat{V}\rangle}{N}=\frac{\rho_{0}}{2} \int d^{2} r_{12} v\left(r_{12}\right)\left[g\left(\mathbf{r}_{12}\right)-1\right],
$$

where $v\left(r_{12}\right)$ can have any reasonable form; in particular, it can take the form of any of the potentials shown in Eqs. (6) and (7). Because the interaction potentials are centrally symmetric, the above formula can be rewritten in the simpler form

$$
E_{\alpha}=\frac{\rho_{0}}{2}(2 \pi) \int_{0}^{\infty} d r_{12} r_{12} v\left(r_{12}\right)\left[\bar{g}\left(r_{12}\right)-1\right],
$$

where $\bar{g}\left(r_{12}\right)$ is the angle-averaged pair distribution function

$$
\bar{g}\left(r_{12}\right)=\int_{0}^{2 \pi} \frac{\mathrm{d} \theta}{2 \pi} g\left(\mathbf{r}_{12}\right) .
$$

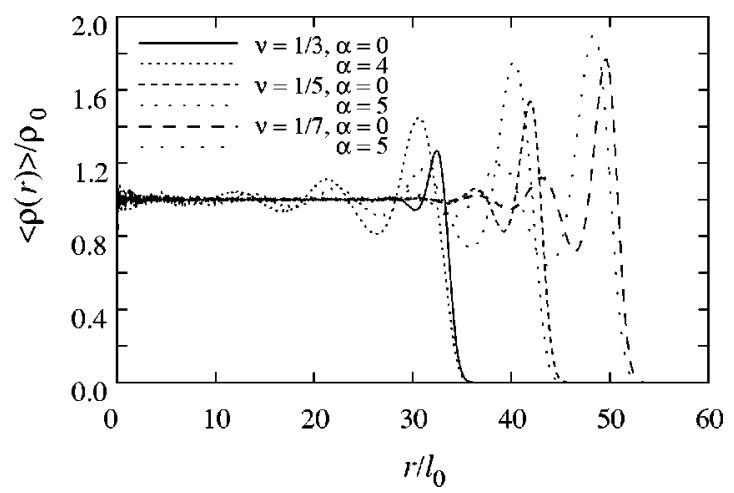

FIG. 1. Angle-averaged single-particle density, $\bar{\rho}(r)$, for $N$ $=196$ electrons and filling factors $\nu=1 / 3,1 / 5$, and $1 / 7$. We show the results for the isotropic cases $(\alpha=0)$ and, for a large $\alpha$ (the oscillations observed in this case are discussed in Sec. V). Here $r$ is the distance from the center of the disk. 

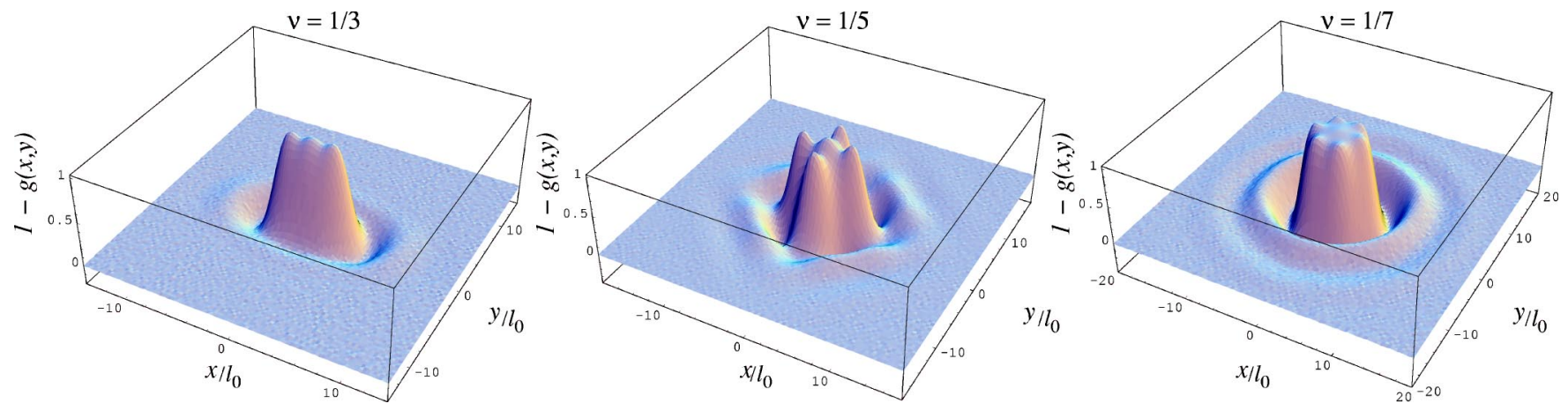

FIG. 2. Pair-correlation function $g(\mathbf{r})$ for $\nu=1 / 3, \alpha=2$ (left panel), $\nu=1 / 5, \alpha=3$ (center panel), $\nu=1 / 7, \alpha=3$ (right panel). Note the discrete rotational symmetry of each state.

For specific cases [as for the $v_{Z D S}\left(r_{12}\right)$ potential, which has strongly oscillatory behavior in real space, making the numerical calculations very unstable and precarious] a corresponding formula that uses the static structure factor was employed:

$$
E_{\alpha}=\frac{1}{2} \frac{1}{(2 \pi)} \int_{0}^{\infty} d q q \tilde{v}(q)[\bar{S}(q)-1] .
$$

In this case $\tilde{v}(q)$ is the $2 \mathrm{D}$ Fourier transform of the interaction potential, and we also define the angle-averaged static structure factor $\bar{S}(q)=\int_{0}^{2 \pi} \mathrm{d} \theta_{q} /(2 \pi) S(\mathbf{q})$. The use of the static structure factor has the added advantage of allowing the calculation of the correlation energies in all LL's from a single determination of the pair-correlation function in the LLL, ${ }^{18-20}$

$$
E_{\alpha}^{(L)}=\frac{1}{2} \int_{0}^{\infty} \frac{d q}{(2 \pi)} q \tilde{v}(q)\left[L_{L}\left(\frac{q^{2}}{2}\right)\right]^{2}[\bar{S}(q)-1],
$$

where $L_{L}(x)$ are Laguerre polynomials and $L$ corresponds to the LL index.

As in any MC calculation using the the Metropolis algorithm, ${ }^{29}$ the expectation value of any [position dependent, e.g., $\rho(\mathbf{r})]$ operator can be computed by averaging the local value of the operator over a large number of electronic configurations generated from the probability distribution $P$ $\propto\left|\Psi_{1 / m}^{\alpha}\right|^{2}$. In a MC attempt, one electron is moved to a new position $\mathbf{r}_{\text {trial }}=\mathbf{r}_{i}+\Delta_{i}$, where $\Delta_{i}$ is a random vector in some domain. If the probability ratio, $P\left(\mathbf{r}_{\text {trial }}\right) / P\left(\mathbf{r}_{i}\right)$ is larger than a random number uniformly distributed in the $[0,1]$ range then the move is accepted and we let $\mathbf{r}_{i+1}=\mathbf{r}_{\text {trial }}$, otherwise the move is rejected and $\mathbf{r}_{i+1}=\mathbf{r}_{i}$. We adjust the size of the domain over which $\Delta_{i}$ 's vary so that about half of the attempted moves are accepted. Following standard practice, we denote a MC step (MCS) a sequence of steps described above so that every electron in the system has attempted a move (and about half succeed). After a MCS the system is in a state essentially uncorrelated to the previous one and averages are computed for the desired operators. ${ }^{30}$ The results we report were obtained after discarding 100000 "thermalization" MCS's and using between $2 \times 10^{6}$ and $4 \times 10^{7}$ MCS's for averaging purposes on systems of 200-400 electrons.

\section{MONTE CARLO RESULTS AND DISCUSSION}

By using MC methods we studied the possibility of a liquid-crystal state in the LLL for the leading candidate states at filling factors, $\nu=1 / 3,1 / 5$, and $1 / 7$. A trial-wave function as in Eq. (3) was considered and various properties were analyzed as function of the anisotropic parameter $\alpha$. Various interaction potentials were considered for the computation of the correlation energies [see Eqs. (6), (7), (10), (11), (13), (14)], all have in common the fact that they incorporate the effects of finite layer thickness into the quasi-2D electronic system and are essentially identical to Coulomb's for large distances. This choice is motivated by the well-known fact that the finite layer thickness of a real 2D system leads to a
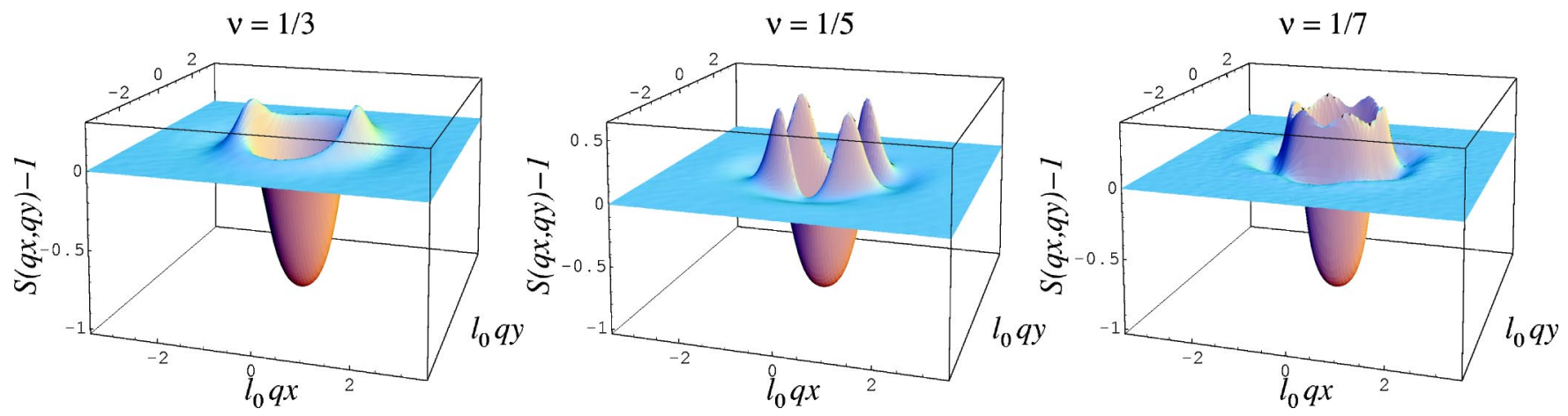

FIG. 3. Static structure factor $S(\mathbf{q})$ for $\nu=1 / 3, \alpha=2$ (left panel), $\nu=1 / 5, \alpha=3$ (center panel), $\nu=1 / 7, \alpha=3$ (right panel). Note the discrete rotational symmetry of each state. 

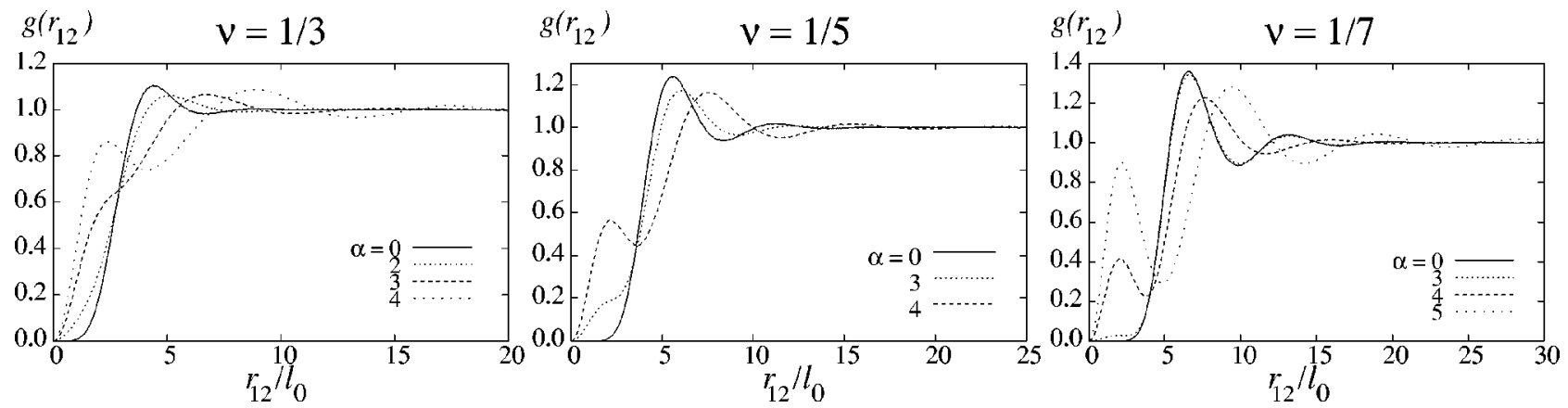

FIG. 4. Angle-averaged pair-correlation function $\bar{g}\left(r_{12}\right)$ for $\nu=1 / 3, \nu=1 / 5$, and $\nu=1 / 7$.

weakening and eventual collapse of the FQHE. ${ }^{31}$ Therefore, when the finite layer thickness (parameter $\lambda$ ) increases as to become larger than the magnetic length, the short-range part of the Coulomb interaction softens and as a result the isotropic FQHE liquid state may become unstable with respect to another state of different nature (a possible new candidate can be the liquid-crystal state considered here, and/or a Wigner crystal).

In Fig. 1 we show a plot of the angle-averaged singleparticle density $\bar{\rho}(r)$ for states of $N=196$ electrons and filling factors of $\nu=1 / 3,1 / 5$, and $1 / 7$. The existence, for $\alpha$ $=0$ of a large region around the center of the disk $(r=0)$ with constant density is an indication that there is bulklike behavior. $^{28}$ Results for moderate values of $\alpha$ are similar to those for $\alpha=0$. For larger $\alpha$ an apparent density fluctuation propagates from the edges to the center making it very difficult to identify a "bulk" region. The existence of this density fluctuation is discussed in detail in Sec. V. We found that values of $\alpha$ acceptable for the purposes of calculating bulklike properties in reasonably sized systems are as follows: $\alpha \lesssim 3$ for $\nu=1 / 3, \alpha \lesssim 4$ for both $\nu=1 / 5$ and $1 / 7$, respectively.

In order to compare the energy of the isotropic Laughlin liquid state with that of an anisotropic liquid-crystal state, we first need an accurate computation of the pair distribution function in terms of the parameter $\alpha$. For the smallest $\alpha$ 's, a number of $N=196$ electrons was sufficient to give a very accurate pair distribution function, whereas as many as 400 electrons were used when $\alpha$ 's became large as to induce sizable oscillations in the density. Figure 2 shows results for

TABLE I. Correlation energy per particle in the LLL (in units of $e^{2} / \epsilon l_{0}$ ) for the liquid-crystal (BRS) states at filling factor $\nu=1 / 3$ as a function of the anisotropy parameter $\alpha$ and quasi-2D layer width $\lambda$. Three forms of the interaction potential were used. The three potentials reduce to the standard Coulomb potential for $\lambda=0$.

\begin{tabular}{|c|c|c|c|c|c|c|c|}
\hline \multicolumn{8}{|c|}{ Interaction potential: $v_{1}\left(r_{12}\right)$} \\
\hline$\alpha$ & $\lambda=0.0$ & $\lambda=0.5$ & $\lambda=1.0$ & $\lambda=1.5$ & $\lambda=2.0$ & $\lambda=2.5$ & $\lambda=3.0$ \\
\hline 0 & -0.4100 & -0.3362 & -0.2776 & -0.2327 & -0.1973 & -0.1700 & -0.1485 \\
\hline 1 & -0.4098 & -0.3353 & -0.2770 & -0.2319 & -0.1970 & -0.1698 & -0.1483 \\
\hline 2 & -0.3961 & -0.3234 & -0.2681 & -0.2257 & -0.1928 & -0.1669 & -0.1464 \\
\hline 3 & -0.3608 & -0.2926 & -0.2449 & -0.2093 & -0.1817 & -0.1597 & -0.1418 \\
\hline 4 & -0.3074 & -0.2435 & -0.2038 & -0.1763 & -0.1554 & -0.1387 & -0.1249 \\
\hline \multicolumn{8}{|c|}{ Interaction potential $v_{2}\left(r_{12}\right)$} \\
\hline$\alpha$ & $\lambda=0.0$ & $\lambda=0.5$ & $\lambda=1.0$ & $\lambda=1.5$ & $\lambda=2.0$ & $\lambda=2.5$ & $\lambda=3.0$ \\
\hline 0 & -0.4100 & -0.3286 & -0.2598 & -0.2107 & -0.1760 & -0.1507 & -0.1315 \\
\hline 1 & -0.4098 & -0.3277 & -0.2593 & -0.2104 & -0.1758 & -0.1505 & -0.1314 \\
\hline 2 & -0.3961 & -0.3162 & -0.2519 & -0.2058 & -0.1727 & -0.1483 & -0.1297 \\
\hline 3 & -0.3608 & -0.2859 & -0.2324 & -0.1936 & -0.1650 & -0.1433 & -0.1264 \\
\hline 4 & -0.3074 & -0.2370 & -0.1950 & -0.1661 & -0.1439 & -0.1265 & -0.1125 \\
\hline \multicolumn{8}{|c|}{ Interaction potential: $v_{Z D S}\left(r_{12}\right)$} \\
\hline$\alpha$ & $\lambda=0.0$ & $\lambda=0.5$ & $\lambda=1.0$ & $\lambda=1.5$ & $\lambda=2.0$ & $\lambda=2.5$ & $\lambda=3.0$ \\
\hline 0 & -0.4100 & -0.3279 & -0.2748 & -0.2381 & -0.2112 & -0.1904 & -0.1738 \\
\hline 1 & -0.4098 & -0.3270 & -0.2741 & -0.2376 & -0.2107 & -0.1900 & -0.1735 \\
\hline 2 & -0.3961 & -0.3160 & -0.2657 & -0.2310 & -0.2053 & -0.1854 & -0.1696 \\
\hline 3 & -0.3608 & -0.2873 & -0.2439 & -0.2138 & -0.1914 & -0.1739 & -0.1597 \\
\hline 4 & -0.3074 & -0.2408 & -0.2054 & -0.1813 & -0.1633 & -0.1491 & -0.1375 \\
\hline
\end{tabular}


TABLE II. Correlation energy per particle in the LLL (in units of $e^{2} / \epsilon l_{0}$ ) for the liquid crystal (BRS) states at filling factor $\nu=1 / 5$ as a function of the anisotropy parameter $\alpha$ and quasi-2D layer width $\lambda$. Three forms of the interaction potential were used. The three potentials reduce to the standard Coulomb potential for $\lambda=0$.

\begin{tabular}{|c|c|c|c|c|c|c|c|}
\hline \multicolumn{8}{|c|}{ Interaction potential: $v_{1}\left(r_{12}\right)$} \\
\hline$\alpha$ & $\lambda=0.0$ & $\lambda=0.5$ & $\lambda=1.0$ & $\lambda=1.5$ & $\lambda=2.0$ & $\lambda=2.5$ & $\lambda=3.0$ \\
\hline 0 & -0.3274 & -0.2811 & -0.2420 & -0.2094 & -0.1825 & -0.1603 & -0.1419 \\
\hline 1 & -0.3273 & -0.2810 & -0.2419 & -0.2094 & -0.1825 & -0.1603 & -0.1419 \\
\hline 2 & -0.3265 & -0.2803 & -0.2413 & -0.2089 & -0.1821 & -0.1600 & -0.1418 \\
\hline 3 & -0.3121 & -0.2674 & -0.2312 & -0.2014 & -0.1767 & -0.1563 & -0.1392 \\
\hline 4 & -0.2775 & -0.2362 & -0.2064 & -0.1829 & -0.1635 & -0.1472 & -0.1333 \\
\hline 5 & -0.2216 & -0.1836 & -0.1601 & -0.1432 & -0.1296 & -0.1181 & -0.1081 \\
\hline \multicolumn{8}{|c|}{ Interaction potential: $v_{2}\left(r_{12}\right)$} \\
\hline$\alpha$ & $\lambda=0.0$ & $\lambda=0.5$ & $\lambda=1.0$ & $\lambda=1.5$ & $\lambda=2.0$ & $\lambda=2.5$ & $\lambda=3.0$ \\
\hline 0 & -0.3274 & -0.2743 & -0.2365 & -0.2086 & -0.1873 & -0.1704 & -0.1566 \\
\hline 1 & -0.3273 & -0.2743 & -0.2365 & -0.2086 & -0.1873 & -0.1704 & -0.1566 \\
\hline 2 & -0.3265 & -0.2767 & -0.2303 & -0.1928 & -0.1641 & -0.1422 & -0.1251 \\
\hline 3 & -0.3121 & -0.2639 & -0.2215 & -0.1870 & -0.1603 & -0.1396 & -0.1233 \\
\hline 4 & -0.2775 & -0.2329 & -0.1997 & -0.1730 & -0.1513 & -0.1338 & -0.1196 \\
\hline 5 & -0.2216 & -0.1801 & -0.1561 & -0.1378 & -0.1222 & -0.1088 & -0.0975 \\
\hline \multicolumn{8}{|c|}{ Interaction potential: $v_{Z D S}\left(r_{12}\right)$} \\
\hline$\alpha$ & $\lambda=0.0$ & $\lambda=0.5$ & $\lambda=1.0$ & $\lambda=1.5$ & $\lambda=2.0$ & $\lambda=2.5$ & $\lambda=3.0$ \\
\hline 0 & -0.3274 & -0.2743 & -0.2365 & -0.2086 & -0.1873 & -0.1704 & -0.1566 \\
\hline 1 & -0.3273 & -0.2743 & -0.2365 & -0.2086 & -0.1873 & -0.1704 & -0.1566 \\
\hline 2 & -0.3265 & -0.2736 & -0.2359 & -0.2082 & -0.1869 & -0.1701 & -0.1563 \\
\hline 3 & -0.3121 & -0.2615 & -0.2265 & -0.2006 & -0.1807 & -0.1648 & -0.1519 \\
\hline 4 & -0.2775 & -0.2324 & -0.2037 & -0.1825 & -0.1659 & -0.1525 & -0.1414 \\
\hline 5 & -0.2216 & -0.1819 & -0.1598 & -0.1439 & -0.1313 & -0.1211 & -0.1124 \\
\hline
\end{tabular}

the pair distribution function, $g(\mathbf{r})$, for the $\nu=1 / 3, \alpha=2$ nematic, $\nu=1 / 5, \alpha=3$ tetratic, and $\nu=1 / 7, \alpha=3$ hexatic. Each MC simulation involved $4 \times 10^{7}$ MCS's and ca. 400 electrons. Figure 3 shows the corresponding static structure factors $S(\mathbf{q})$ obtained from $g(\mathbf{r})$ using Eq. (9).

Since the angle-averaged $\bar{g}\left(r_{12}\right)$ is sufficient for the determination of the energy, we averaged it (at significant savings in computer time) for various combinations of filling factor $\nu$, and anisotropy parameter $\alpha$. Figure 4 shows some of our results for 196-400 electrons.

At all filling factors that we considered, we noted that $\bar{g}\left(r_{12}\right)$ changes very little when parameter $\alpha$ is small (e.g., $1)$. Only for larger $\alpha$ 's ( $\gtrsim 2)$ sizable changes take effect. In view of this behavior, we anticipate that the energy differences between the isotropic liquid state $(\alpha=0)$ and the anisotropic liquid-crystal state with small anisotropy parameters $(\alpha=1)$ will be quite small. In fact, the calculation of energy differences between these states and the isotropic state are comparable to the estimated accuracy of our energy calculations. However, since the energy differences for larger $\alpha$ 's show a definite tendency in all cases, we believe that the results are, significantly reliable (since the statistical uncertainty on any MC calculation is systematic, the energy differences may be even more accurate than the absolute energies).

Tables I-III present the results for the calculation of the
LLL correlation energies obtained by means of Eqs. (11) or (13), using the angle-averaged pair-correlation functions (or static structure factors) for the three different forms of the interaction potential for a variety of quasi-2D layer widths $\lambda$ [see Eqs. (6) and (7)]. When $\lambda=0$ all interaction potentials reduce to the Coulomb potential and in the case of the $v_{Z D S}\left(r_{12}\right)$ potential we note that $b=1.5 / \lambda$. Results for filling factors $\nu=1 / 3,1 / 5$, and $1 / 7$ of the LLL [for the potential $\left.v_{1}\left(r_{12}\right)\right]$ are also presented in Fig. 5. The results suggest that, in the LLL, for all the interaction potentials under consideration, a uniform liquid state is energetically more favorable than the liquid-crystal state. For small values of $\alpha \in(0$, $\approx 2]$, the liquid-crystal states have an energy only slightly above the Laughlin liquid states $(\alpha=0)$, however for larger $\alpha$ 's this difference increases.

Similar results are obtained in the first excited LL $[L=1$ in Eq. (14), we omit the results for brevity]. For all forms of the interaction potential considered here, the correlation energy for anisotropic states is higher, once again leaving the Laughlin state stable. However, it is interesting to note that for the second excited LL $(L=2)$ the situation changes for the nematic states at $\nu=1 / 3$ of the valence LL, where anisotropic states become energetically favorable. Table IV shows the results for the energies, $E_{\alpha}$ and energy differences, $\Delta E_{\alpha} \equiv E_{\alpha}-E_{0}$ (also shown in Fig. 6) between anisotropic states $(\alpha \neq 0)$ and the isotropic state $(\alpha=0)$ for filling factor 
TABLE III. Correlation energy per particle in the LLL (in units of $e^{2} / \epsilon l_{0}$ ) for the liquid crystal (BRS) states at filling factor $\nu=1 / 7$ as a function of the anisotropy parameter $\alpha$ and quasi-2D layer width $\lambda$. Three forms of the interaction potential were used. The three potentials reduce to the standard Coulomb potential for $\lambda=0$.

\begin{tabular}{|c|c|c|c|c|c|c|c|}
\hline \multicolumn{8}{|c|}{ Interaction potential: $v_{1}\left(r_{12}\right)$} \\
\hline$\alpha$ & $\lambda=0.0$ & $\lambda=0.5$ & $\lambda=1.0$ & $\lambda=1.5$ & $\lambda=2.0$ & $\lambda=2.5$ & $\lambda=3.0$ \\
\hline 0 & -0.2827 & -0.2491 & -0.2198 & -0.1944 & -0.1727 & -0.1541 & -0.1383 \\
\hline 1 & -0.2827 & -0.2491 & -0.2198 & -0.1944 & -0.1727 & -0.1541 & -0.1383 \\
\hline 2 & -0.2826 & -0.2491 & -0.2198 & -0.1944 & -0.1727 & -0.1541 & -0.1383 \\
\hline 3 & -0.2807 & -0.2473 & -0.2184 & -0.1933 & -0.1719 & -0.1536 & -0.1379 \\
\hline 4 & -0.2492 & -0.2185 & -0.1945 & -0.1745 & -0.1573 & -0.1425 & -0.1296 \\
\hline 5 & -0.1917 & -0.1643 & -0.1467 & -0.1334 & -0.1223 & -0.1124 & -0.1035 \\
\hline \multicolumn{8}{|c|}{ Interaction potential: $v_{2}\left(r_{12}\right)$} \\
\hline$\alpha$ & $\lambda=0.0$ & $\lambda=0.5$ & $\lambda=1.0$ & $\lambda=1.5$ & $\lambda=2.0$ & $\lambda=2.5$ & $\lambda=3.0$ \\
\hline 0 & -0.2827 & -0.2470 & -0.2123 & -0.1821 & -0.1576 & -0.1382 & -0.1227 \\
\hline 1 & -0.2827 & -0.2470 & -0.2124 & -0.1822 & -0.1577 & -0.1383 & -0.1227 \\
\hline 2 & -0.2826 & -0.2470 & -0.2123 & -0.1821 & -0.1576 & -0.1382 & -0.1227 \\
\hline 3 & -0.2807 & -0.2452 & -0.2110 & -0.1813 & -0.1571 & -0.1378 & -0.1225 \\
\hline 4 & -0.2492 & -0.2164 & -0.1894 & -0.1659 & -0.1460 & -0.1297 & -0.1162 \\
\hline 5 & -0.1917 & -0.1621 & -0.1443 & -0.1295 & -0.1160 & -0.1040 & -0.0936 \\
\hline \multicolumn{8}{|c|}{ Interaction potential: $v_{Z D S}\left(r_{12}\right)$} \\
\hline$\alpha$ & $\lambda=0.0$ & $\lambda=0.5$ & $\lambda=1.0$ & $\lambda=1.5$ & $\lambda=2.0$ & $\lambda=2.5$ & $\lambda=3.0$ \\
\hline 0 & -0.2827 & -0.2436 & -0.2141 & -0.1914 & -0.1735 & -0.1591 & -0.1471 \\
\hline 1 & -0.2827 & -0.2437 & -0.2141 & -0.1915 & -0.1736 & -0.1591 & -0.1472 \\
\hline 2 & -0.2826 & -0.2436 & -0.2141 & -0.1914 & -0.1735 & -0.1591 & -0.1471 \\
\hline 3 & -0.2807 & -0.2419 & -0.2128 & -0.1904 & -0.1727 & -0.1584 & -0.1465 \\
\hline 4 & -0.2492 & -0.2147 & -0.1906 & -0.1720 & -0.1572 & -0.1450 & -0.1348 \\
\hline 5 & -0.1917 & -0.1627 & -0.1454 & -0.1323 & -0.1216 & -0.1126 & -0.1050 \\
\hline
\end{tabular}

$\nu=1 / 3$ in the second excited LL $(L=2)$ obtained from potential $v_{1}\left(r_{12}\right)$ (the results are quite similar for the other two forms of the potential). These results are generally consistent to what we found in the past using the hypernetted-chain approximation. ${ }^{18,19}$

A conclusion can be derived from the above results: generally speaking the isotropic states seem to be energetically favorable, with the exception of the nematic state in the second excited LL. The explanation for this is simple: in the LLL the electron packets are simple Gaussians, and it is clear that the best way to minimize their Coulomb repulsion is by placing the vortices responsible for the $\mathrm{CF}$ transformation ${ }^{4,5}$ precisely at the location of the electron themselves $(\alpha=0)$. In higher LL's, the wavepackets take a more "ringlike" shape, and a finite $\alpha$ permits a more optimal distribution of charge for the nematic case (but not for either the tetratic or hexatic).

\section{UNDERLYING CHARGE-DENSITY WAVE IN THE ANISOTROPIC 2DOCP'S}

In view of the appearance of considerable density variations in our MC simulations for larger values of $\alpha$ we investigated the possible existence of an underlying CDW for the
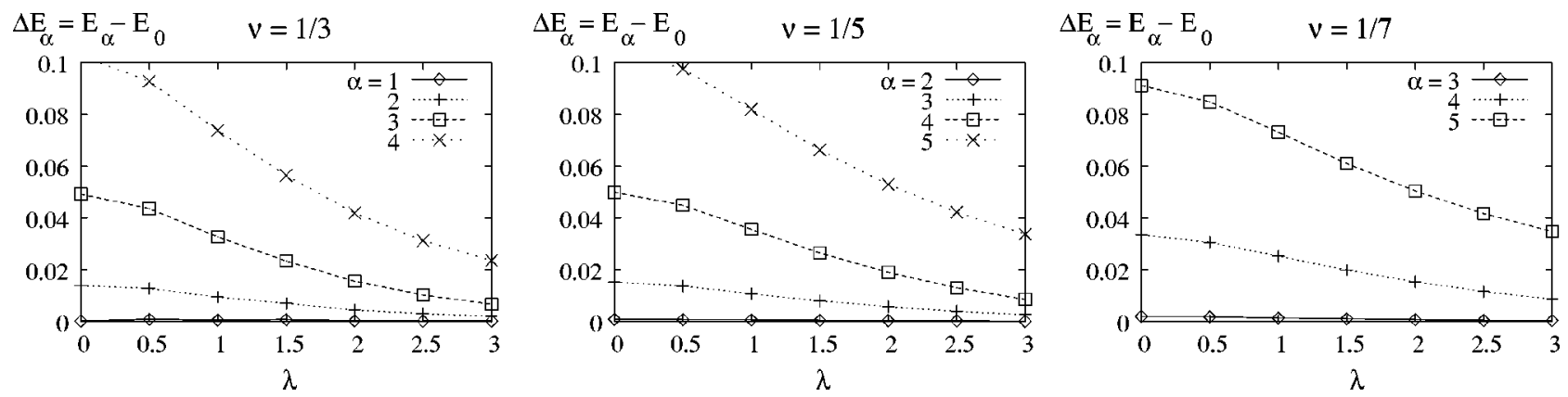

FIG. 5. Energy difference between anisotropic states and the isotropic state $(\alpha=0) \Delta E_{\alpha} \equiv E_{\alpha}-E_{0}$ for filling factors $\nu=1 / 3,1 / 5$, and $1 / 7$ in the LLL. These results correspond to the interaction potential $v_{1}\left(r_{12}\right)$ and are plotted as function of the quasi-2D layer thickness $\lambda$. 
TABLE IV. Correlation energy per particle in the second excited LL, $L=2$, (in units of $e^{2} / \epsilon l_{0}$ ) for the liquid crystal (BRS) states at filling factor $\nu=1 / 3$ as a function of the anisotropy parameter $\alpha$ and quasi-2D layer width $\lambda$. The form $v_{1}\left(r_{12}\right)$ for the interaction potential was used.

\begin{tabular}{lccccccc}
\hline \hline \multicolumn{7}{c}{ Interaction potential: $v_{1}\left(r_{12}\right)$} \\
\hline$\alpha$ & $\lambda=0.0$ & $\lambda=0.5$ & $\lambda=1.0$ & $\lambda=1.5$ & $\lambda=2.0$ & $\lambda=2.5$ & $\lambda=3.0$ \\
0 & -0.2642 & -0.2139 & -0.1872 & -0.1662 & -0.1485 & -0.1335 & -0.1207 \\
1 & -0.2653 & -0.2146 & -0.1875 & -0.1663 & -0.1486 & -0.1335 & -0.1208 \\
2 & -0.2693 & -0.2169 & -0.1881 & -0.1663 & -0.1483 & -0.1333 & -0.1206 \\
3 & -0.2708 & -0.2158 & -0.1852 & -0.1631 & -0.1455 & -0.1310 & -0.1188 \\
\hline \hline
\end{tabular}

liquid crystalline states of Eq. (3). For this purpose it is useful to consider, once again, the 2DOCP analog system. Whereas considerable effort has been dedicated (and a consequent vast knowledge has been achieved) in the past to the treatment of the standard isotropic plasma (see, e.g., Refs. 10,32,33 and 26), little has been pursued for a system with anisotropic interactions, e.g., quadrupolar terms.

Consider the classical distribution function (note, in this section we work in units of the magnetic length $l_{0}$ )

$$
\begin{gathered}
\left|\Psi_{1 / m}\right|^{2} \propto e^{-\beta V}, \quad \text { where } \quad-\beta V=2 \sum_{i<j}^{N}\left[\ln \left|z_{i}-z_{j}\right|\right. \\
\left.+\sum_{\mu=1}^{m-1}\left|z_{i}-z_{j}-\alpha_{\mu}\right|\right]-\frac{1}{2} \sum_{k=1}^{N}\left|z_{k}\right|^{2}
\end{gathered}
$$

where, as before, $\alpha_{\mu}=\alpha e^{i \theta_{\mu}}, \theta_{\mu}=2 \pi(\mu-1) /(m-1)$, and $\mu \in\{1,2, \ldots,(m-1)\}$. This potential energy corresponds to an "electrostatic potential" which is solution of a modified Poisson's equation

$$
\nabla^{2}[\beta \phi(\mathbf{r})]=-4 \pi\left[\rho(\mathbf{r})+\sum_{\mu=1}^{m-1} \rho\left(\mathbf{r}-\vec{\alpha}_{\mu}\right)\right]+4 \pi m \rho_{0}
$$

where $\vec{\alpha}_{\mu}=\alpha\left(\cos \theta_{\mu}, \sin \theta_{\mu}\right)$,

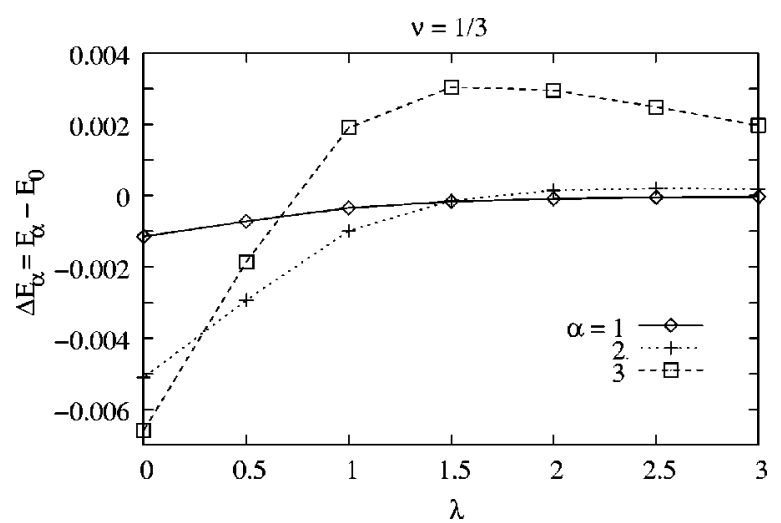

FIG. 6. Energy difference between anisotropic states and the isotropic state $(\alpha=0) \Delta E_{\alpha} \equiv E_{\alpha}-E_{0}$ for filling factor $\nu=1 / 3$ in the second excited valence LL $(L=2)$. These results correspond to the interaction potential form $v_{1}\left(r_{12}\right)$ and are plotted as function of the quasi-2D layer thickness $\lambda$.

$$
\rho(\mathbf{r})=\sum_{i=1}^{N} \delta\left(\mathbf{r}-\mathbf{r}_{i}\right)
$$

and $\rho_{0}=1 /(2 \pi m)$ is a neutralizing density.

Consider now the potential $V$ generated by the addition of some charge $\delta \rho(\mathbf{r})$. This will cause a redistribution of the particles that form the plasma, inducing a density change [see the discussion related to the definition of the paircorrelation function, Eq. (8)],

$$
\rho_{\text {ind }}(\mathbf{r})=\int d^{2} r^{\prime} \rho_{0}\left[g\left(\mathbf{r}-\mathbf{r}^{\prime}\right)-1\right] \delta \rho\left(\mathbf{r}^{\prime}\right)
$$

The total charge, in reciprocal space, is therefore given by [see Eq. (9)]

$$
\tilde{\rho}_{t o t}(\mathbf{k})=S(\mathbf{k}) \widetilde{\delta \rho}(\mathbf{k}),
$$

leading to a total potential

$$
\beta \widetilde{\phi}(\mathbf{k})=\frac{4 \pi S(\mathbf{k})}{k^{2}}\left[1+\sum_{\mu=1}^{m-1} e^{i \vec{\alpha}_{\mu} \cdot \mathbf{k}}\right] \widetilde{\delta \rho(\mathbf{k})} .
$$

This result neglects second-order corrections in the distribution functions and is, therefore, commonly referred to as the theory of linear screening.

It is now interesting to investigate whether this potential allows for the formation of underlying CDW's in the 2DOCP. Assuming small variations from a uniform state, we allow for the particle density to vary from point to point according to

$$
\rho(\mathbf{r})=\rho_{0}+\rho_{1} \cos (\mathbf{q} \cdot \mathbf{r}),
$$

where $\mathbf{q}$ is the wave vector of the $\mathrm{CDW}$ and $\rho_{1} \ll \rho_{0}$. The $\mathcal{O}\left[\rho_{1}^{2}\right]$ "excess energy" 10 per unit area is given by

$$
\frac{\beta u^{e x c}}{\rho_{1}^{2}}=\frac{1}{2} \frac{2 \pi S(\mathbf{q})}{q^{2}}\left[1+\sum_{\mu=1}^{m-1} e^{i \vec{\alpha}_{\mu} \cdot \mathbf{q}}\right] \text {. }
$$

It is evident that the charge neutrality sum rule $\left[S(\mathbf{q}) \propto q^{2}\right.$ for $\left.q \rightarrow 0^{10,25,26}\right]$ guarantees the elimination of the singularity at $q=0$ leading to screening of the interaction. More interesting, however, is the fact that the excess energy becomes negative for a variety of wave vectors when $\alpha \neq 0$. If we write Eq. (22) explicitly for the various states considered in this paper: 

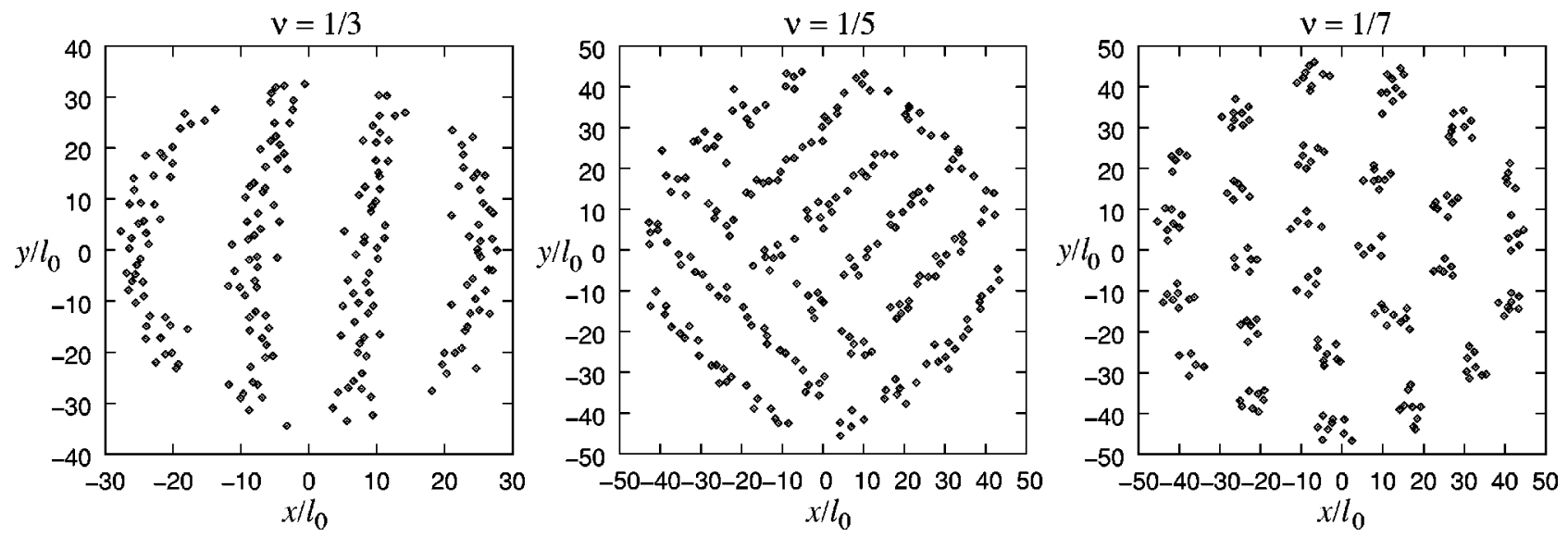

FIG. 7. Typical electron configurations for a nematic $(\nu=1 / 3, \alpha=7$, left panel), tetratic $(\nu=1 / 5, \alpha=8$, center panel), and hexatic $(\nu=1 / 7, \alpha=10$, right panel). Note the formation of a CDW's with one, two, and three different directors.

$$
\operatorname{nematic}(\nu=1 / 3): \frac{1}{2} \frac{2 \pi S(\mathbf{q})}{q^{2}}\left[1+2 \cos \left(\alpha q_{x}\right)\right]
$$

tetratic $(\nu=1 / 5): \frac{1}{2} \frac{2 \pi S(\mathbf{q})}{q^{2}}\left[1+2 \cos \left(\alpha q_{x}\right)+2 \cos \left(\alpha q_{y}\right)\right]$

$$
\begin{aligned}
\operatorname{hexatic}(\nu= & 1 / 7): \frac{1}{2} \frac{2 \pi S(\mathbf{q})}{q^{2}}\left[1+2 \cos \left(\alpha q_{x}\right)\right. \\
& +2 \cos \left[\alpha\left(-\frac{1}{2} q_{x}+\frac{\sqrt{3}}{2} q_{y}\right)\right] \\
& \left.+2 \cos \left[\alpha\left(-\frac{1}{2} q_{x}-\frac{\sqrt{3}}{2} q_{y}\right)\right]\right],
\end{aligned}
$$

We can see that the most important configurations (those that make the potential $\beta V$ minimum and maximize their probability) correspond to charge-density waves with wave vectors in the neighborhood of ${ }^{34} \alpha \mathbf{q} / \pi \simeq\{(1,0),(-1,0)\}$ for the nematic, $\alpha \mathbf{q} / \pi \simeq\{(1,1),(1,-1),(-1,1),(-1,-1)\}$ for the tetratic, and $\alpha \mathbf{q} / \pi \simeq\left\{\left(\frac{4}{3}, 0\right),\left(-\frac{4}{3}, 0\right),\left(\frac{2}{3}, 2 / \sqrt{3}\right),\left(\frac{2}{3},-2 / \sqrt{3}\right)\right.$, $\left.\left(-\frac{2}{3}, 2 / \sqrt{3}\right),\left(-\frac{2}{3},-2 / \sqrt{3}\right)\right\}$ for the hexatic. This should produce a unidirectional CDW (a layered system, or smectic) underlying the nematic, with a characteristic wavelength $\lambda$ $\simeq 2 \alpha$; a square lattice tilted $45^{\circ}$ with lattice constant $a$ $\simeq \sqrt{2} \alpha$, and a triangular lattice with triangle side $a=\sqrt{3} \alpha$. Figure 7 depicts typical configurations during MC simulations with large $\alpha$ 's. The characteristic CDW's have periods very close to those predicted above.

One should note that these underlying CDW's are extremely soft and fluctuations will render them invisible in the thermodynamic and ergodic limits. In our simulations, however, their effects are perceptible (see, e.g., Fig. 1) for large values of the anisotropy parameter $\alpha$ because of phase locking at the boundaries. A detailed study of the fluctuations of these CDW's will be published elsewhere. ${ }^{35}$

\section{CONCLUSIONS}

In conclusion, we have investigated the possibility of liquid-crystal states in quasi-two-dimensional electron systems in strong magnetic fields. We considered translation invariant yet anisotropic states at filling factors $\nu=1 / 3,1 / 5$, and $1 / 7$ of the lowest $(L=0)$, first excited $(L=1)$ and second excited $(L=2)$ LL's. We found that the anisotropic states possess an underlying CDW along directors with the same symmetry group of the proposed state but these CDW's are "washed-out" by fluctuations. We applied MC methods to calculate the (angle-dependent) pair-correlation function and static structure factors for these states, which have permitted us to calculate the correlation energies for a variety of reasonable generalizations of the Coulomb potential that take into consideration the finite width of the quasi-2D layer. For all states and potentials under consideration the isotropic Laughlin state is found to be energetically favorable in the lowest and first excited LL, whereas we find an instability of the $\nu=1 / 3$ nematic state in the second excited LL.

\section{ACKNOWLEDGMENTS}

We would like to thank A.T. Dorsey, A.H. MacDonald, E. Fradkin, M. Fogler, and J. MacCollough for useful discussions. Acknowledgment is made to the University of Missouri Research Board and to the Donors of the Petroleum Research Fund, administered by the American Chemical Society, for support of this research. One of the authors (O.C.) would like to acknowledge R. Wilkins and K. Kerby for their hospitality during summer 2003. Part of the work was supported by NASA-CARR at Prairie View A\&M University. 
${ }^{1}$ R.B. Laughlin, Phys. Rev. Lett. 50, 1395 (1983).

${ }^{2}$ D.C. Tsui, H.L. Stormer, and A.C. Gossard, Phys. Rev. Lett. 48, 1559 (1982).

${ }^{3}$ In Perspectives in Quantum Hall effects, edited by S. Das Sarma and A. Pinczuk (Wiley, New York, 1996).

${ }^{4}$ In Composite Fermions, edited by O. Heinonen (World Scientific, New York, 1998).

${ }^{5}$ J. Jain, Phys. Today 53 (4), 39 (2000).

${ }^{6}$ E.E. Mendez, M. Heiblum, L.L. Chang, and L. Esaki, Phys. Rev. B 28, 4886 (1983).

${ }^{7}$ P.K. Lam and S.M. Girvin, Phys. Rev. B 30, 473 (1984); D. Levesque, J.J. Weis, and A.H. MacDonald, ibid. 30, 1056 (1984); K. Esfarjani and S.T. Chui, ibid. 42, 10758 (1984); X. Zhu and S.G. Louie, ibid. 52, 5863 (1990).

${ }^{8}$ K. Yang, F.D.M. Haldane, and E.H. Rezayi, Phys. Rev. B 64, 081301 (2001).

${ }^{9}$ The wave function for $m=1$ corresponds to the integer QHE, and is also a pure Slater determinant of states in the LLL.

${ }^{10}$ J.M. Caillol, D. Levesque, J.J. Weis, and J.P. Hansen, J. Stat. Phys. 28, 325 (1982).

${ }^{11}$ D. Levesque, J.J. Weis, and A.H. MacDonald, Phys. Rev. B 30, 1056 (1984).

${ }^{12}$ J.M. Kosterlitz and D.J. Thouless, J. Phys. C 6, 1181 (1973).

${ }^{13}$ B.I. Halperin and D.R. Nelson, Phys. Rev. Lett. 41, 121 (1978).

${ }^{14}$ A.P. Young, Phys. Rev. B 19, 1855 (1979).

${ }^{15}$ M.P. Lilly, K.B. Cooper, J.P. Eisenstein, L.N. Pfeiffer, and K.W. West, Phys. Rev. Lett. 82, 394 (1999); R.R. Du, D.C. Tsui, H.L. Stormer, L.N. Pfeiffer, K.W. Baldwin, and K.W. West, Solid State Commun. 109, 389 (1999); M. Shayegan, H.C. Manoharan, S.J. Papadakis, E.P. DePoortere, Physica E 6, 40 (2000).

${ }^{16}$ E. Fradkin and S.A. Kivelson, Phys. Rev. B 59, 8065 (1999); S.A. Kivelson, E. Fradkin, and V.J. Emery, Nature (London) 393, 550 (1998).
${ }^{17}$ C. Wexler and A.T. Dorsey, Phys. Rev. B 64, 115312 (2001).

${ }^{18}$ O. Ciftja and C. Wexler, Phys. Rev. B 65, 045306 (2002).

${ }^{19}$ O. Ciftja and C. Wexler, Phys. Rev. B 65, 205307 (2002).

${ }^{20}$ C. Wexler and O. Ciftja, J. Phys.: Condens. Matter 14, 3705 (2002).

${ }^{21}$ W. Pan, H.L. Stormer, D.C. Tsui, L.N. Pfeiffer, K.W. Baldwin, and K.W. West, Phys. Rev. Lett. 88, 176802 (2002).

${ }^{22}$ A.J. Schmidt, O. Ciftja, and C. Wexler, Phys. Rev. B 67, 155315 (2003).

${ }^{23}$ C.M. Lapilli, O. Ciftja, and C. Wexler (unpublished).

${ }^{24}$ K. Musaelian and R. Joynt, J. Phys.: Condens. Matter 8, L105 (1996).

${ }^{25}$ S.M. Girvin, A.H. MacDonald, and P.M. Platzman, Phys. Rev. B 33, 2481 (1986).

${ }^{26}$ K.I. Golden and D. Merlini, Phys. Rev. A 16, 438 (1977).

${ }^{27}$ F.C. Zhang and S. Das Sarma, Phys. Rev. B 33, 2903 (1986).

${ }^{28}$ O. Ciftja and C. Wexler, Phys. Rev. B 67, 075304 (2003).

${ }^{29}$ N. Metropolis, A.W. Rosenbluth, M.N. Rosenbluth, A.M. Teller, and E. Teller, J. Chem. Phys. 21, 1087 (1953).

${ }^{30}$ We have verified that the states are essentially uncorrelated after one MCS by observing that the dependence of the random noise in the averaged operators behaves essentially like $N_{M C S}^{-1 / 2}$, where $N_{M C S}$ is the number of MCS's performed.

${ }^{31}$ S. He, F.C. Zhang, X.C. Xie, and S. Das Sarma, Phys. Rev. B 42, 11376 (1990).

${ }^{32}$ C. Deutsch, H.E. Dewitt, and Y. Furutani, Phys. Rev. A 20, 2631 (1979).

${ }^{33}$ J.P. Hansen and D. Levesque, J. Phys. C 14, L603 (1981).

${ }^{34}$ We should note that, except for extremely large $\alpha$ 's, the static structure factor $S(\mathbf{q})$ actual dependence on $\mathbf{q}$ slightly modifies the following results (by about 10\%).

${ }^{35}$ C.M. Lapilli and C. Wexler (unpublished). 